\title{
3D NMR spectroscopy for resonance assignment and structure elucidation of proteins under MAS: novel pulse schemes and sensitivity considerations ${ }^{\text {in }}$
}

\author{
Henrike Heise*, Karsten Seidel, Manuel Etzkorn, Stefan Becker, Marc Baldus* \\ Department for NMR-based Structural Biology, Max-Planck-Institute for Biophysical Chemistry, Am Fassberg 11, D-37077 Göttingen, Germany
}

Received 13 September 2004; revised 15 November 2004

Available online 15 December 2004

\begin{abstract}
Two types of 3D MAS NMR experiments are introduced, which combine standard (NC,CC) transfer schemes with $\left({ }^{1} \mathrm{H},{ }^{1} \mathrm{H}\right)$ mixing to simultaneously detect connectivities and structural constraints of uniformly ${ }^{15} \mathrm{~N}$, ${ }^{13} \mathrm{C}$-labeled proteins with high spectral resolution. The homonuclear CCHHC and CCC experiments are recorded with one double-quantum evolution dimension in order to avoid a cubic diagonal in the spectrum. Depending on the second transfer step, spin systems or proton-proton contacts can be determined with reduced spectral overlap. The heteronuclear NHHCC experiment encodes NH-HC proton-proton interactions, which are indicative for the backbone conformation of the protein. The third dimension facilitates the identification of the amino acid spin system. Experimental results on $\mathrm{U}-\left[{ }^{15} \mathrm{~N},{ }^{13} \mathrm{C}\right]$ valine and $\mathrm{U}-\left[{ }^{15} \mathrm{~N},{ }^{13} \mathrm{C}\right]$ ubiquitin demonstrate their usefulness for resonance assignments and for the determination of structural constraints. Furthermore, we give a detailed analysis of alternative multidimensional sampling schemes and their effect on sensitivity and resolution.
\end{abstract}

(C) 2004 Elsevier Inc. All rights reserved.

Keywords: 3D MAS NMR; Sensitivity; Alternative sampling schemes; Proton-proton contacts; Resonance assignment

\section{Introduction}

Solid-state MAS [1] NMR spectroscopy has become a useful method for structural studies on proteins that can be neither solubilized nor crystallized, as is often the case for membrane-bound proteins, protein aggregates, and fibrils [2-6]. Investigation of the $3 \mathrm{D}$ protein structure requires a large number of structural constraints, which ideally should be available from one or a limited set of samples. Therefore, much effort has recently been put into the study of uniformly or multiply

\footnotetext{
Presented in part at the 45th ENC Conference, Asilomar, CA, April 18-23, 2004.

* Corresponding authors. Fax: +495512012202.

E-mail addresses: hehe@nmr.mpibpc.mpg.de (H. Heise), maba@ mpibpc.mpg.de (M. Baldus).
}

${ }^{13} \mathrm{C}$ - and ${ }^{15} \mathrm{~N}$-labeled proteins by solid-state MAS NMR spectroscopy.

The first step towards structure elucidation is the assignment of the observable ${ }^{13} \mathrm{C}$ and ${ }^{15} \mathrm{~N}$ resonances to the corresponding nuclei. In the past decade, a variety of dipolar recoupling techniques have been developed (see, for example [7-10]), which are characterized by different bandwidths and transfer characteristics. In larger proteins, the resolution is limited by spectral overlap and multidimensional NMR experiments are mandatory. Different heteronuclear 3D NCC [11-16] and CNC $[17,18]$ experiments have been suggested and have proven to be useful for spectral assignment. For the assignment of proton resonances, ${ }^{1} \mathrm{H}$ evolution periods have been combined with one or two rare-spin (i.e., ${ }^{13} \mathrm{C}$ or ${ }^{15} \mathrm{~N}$ ) detected time domains $[19-21,75]$. Furthermore, a set of $3 \mathrm{D}$ experiments with two high-resolution dimensions $\left({ }^{13} \mathrm{C}\right.$ or $\left.{ }^{15} \mathrm{~N}\right)$ have been designed for the 
determination of multiple torsion angle or distance constraints. These experiments monitor the dephasing of double-quantum coherences or cross-peak signal amplitudes under the combined effect of two dipolar couplings $[22,23]$. In addition, NMR schemes that read out the dependence of the intensity of homonuclear cross-peaks on the spinning frequency or on the amplitude of a weak radio-frequency field under chemical-shift selective dipolar recoupling conditions have been reported $[24,25]$.

As is well known from protein solution-state NMR $[26,27]$, the determination of proton-proton distance constraints provides a rich source for defining secondary and tertiary protein structure by NMR. Because of the limited spectral resolution of ${ }^{1} \mathrm{H}$ MAS NMR spectroscopy, indirect detection schemes for proton-proton interactions have been employed for a long time (see, for example [28-35]). In addition to the study of macromolecular assemblies [36,37], such N/CHHC methods $[33,34]$ were recently used to probe protein-protein interfaces [38], molecular dynamics [39], and to determine the three-dimensional structure of uniformly ${ }^{13} \mathrm{C},{ }^{15} \mathrm{~N}-\mathrm{la}$ beled (poly)peptides under MAS conditions $[34,40]$. With increasing number of residues, spectral overlap can complicate an unequivocal spectral assignment and structural analysis. For this reason, we introduce in the following novel three-dimensional high-resolution MAS NMR experiments, which efficiently combine standard rare-spin (NC,CC) polarization transfer schemes $[7,8,41-46]$ with mixing units that indirectly encode $\left({ }^{1} \mathrm{H},{ }^{1} \mathrm{H}\right)$ contacts with increased resolution. In addition, we present a homonuclear $3 \mathrm{D}$ experiment containing one double-quantum evolution dimension, which is useful for the identification of spin systems and can, under appropriate experimental conditions [47], be applied for sequential resonance assignment of amino acids in uniformly labeled proteins.

One drawback of 3D NMR spectroscopy is the larger time requirement, due to the increased dimensionality of the indirect time space. In solution-state NMR, the minimal total acquisition time is often dictated not by the sensitivity but by the total number of scans required, and a variety of methods for faster acquisition of $N$-dimensional spectra have been developed, which circumvent the sampling of the full $\left(t_{1}, \ldots, t_{N-1}\right)$ space [48-51]. Reduced dimensionality experiments [52] have also been applied in solid-state MAS NMR spectroscopy $[17,53,54]$, as well as nonlinear sampling [55]. However, due to the low sensitivity of solid-state NMR experiments (for example, as a result of multiple polarization transfer steps and the lower gyromagnetic ratios of the rare-spin nuclei), the main requirement for a multidimensional sampling scheme is not the reduction of the total number of scans but rather the maximization of the average signal-to-noise ratio $\left(S / \sigma_{\mathrm{N}}\right)$ per scan. A seemingly attractive approach towards this goal is adapting the sampling protocol in the indirect dimen- sions to the two-dimensional decay curve of the magnetization. In particular, a triangular sampling scheme has been suggested earlier for solution-state NMR [56], where data points are only acquired in the lower triangle of the $\left(t_{1}, t_{2}\right)$ plane (defined by the condition $t_{1} /$ $t_{1 \max }+t_{2} / t_{2 \max } \leqslant 1$ ), the region where the intensity of the exponentially decaying magnetization curve is strongest. A gain of factor 2 in sensitivity compared to acquisition of the full dataset was postulated for long acquisition times, and a similar triangular truncation of 2D data sets was suggested as an alternative apodization method [57]. However, a reduction of the time domain of multidimensional data sets is always compromised by a decrease in resolution. Therefore, effects of data truncation have to be included in the discussion of alternative sampling schemes in order to obtain a fair comparison to traditional sampling schemes. The purpose of this paper is twofold: In Section 2.1, we introduce 3D pulse schemes that are tailored to the detection of proton-proton contacts with enhanced spectral resolution. Second, we present a detailed analysis of alternative sampling schemes and their effects on sensitivity and resolution in Section 2.2. In Section 3, experimental results are shown for $\mathrm{U}-\left[{ }^{15} \mathrm{~N},{ }^{13} \mathrm{C}\right] \mathrm{Val}$ and ubiquitin. The effect of different acquisition schemes on the quality of the spectrum is demonstrated for a $3 \mathrm{D}$ CCHHC spectrum of U-Val.

\section{Methods}

\section{1. $3 D$ pulse sequences}

In Fig. 1A, the pulse scheme for the homonuclear high-resolution 3D NMR experiments is depicted. To avoid a cubic diagonal, the sequence contains one double-quantum (2Q) $t_{1}$ evolution dimension, such that each signal is encoded with at least two frequencies. For double-quantum excitation and reconversion, any homonuclear dipolar recoupling sequence, such as symmetry-based pulse schemes or HORROR (see, for example $[7,8,42,45,46])$, can be used. In order to restrict the frequency-range of the double-quantum dimension to the side chain region of the spectrum, recoupling schemes requiring low RF power, such as HORROR [42] (Fig. 1B) or R $18_{10}^{5}$ or R18 8 (Fig. 1C) [46], are preferable. The coherence pathway indicated in the diagram of Fig. 1A is selected by cycling the RF and receiver phases as given in the figure caption. After reconversion to single-quantum coherence, the second homonuclear mixing can be achieved either by dipolar CC mixing or by transfer via proton-proton interactions. Examples for proton-driven spin-diffusion [41] and longitudinal proton-proton mixing [33] are given in Figs. 1D and E, respectively. Note that proton-mediated magnetization transfer is indicative for short proton-proton con- 

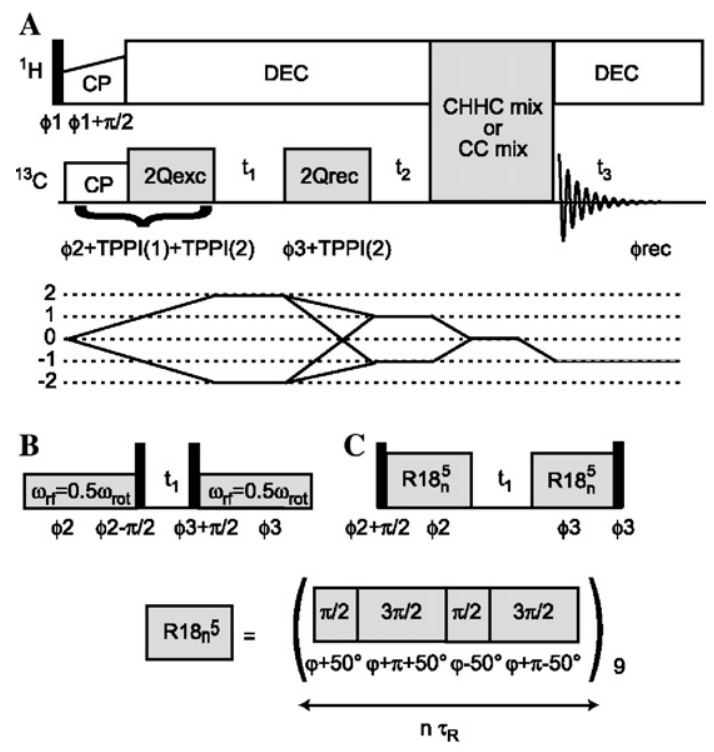

D

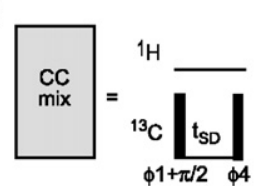

$\mathbf{E}$

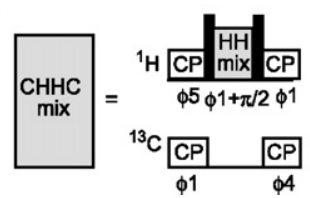

Fig. 1. Pulse sequences for 3D homonuclear correlation experiments in the solid state. Black rectangles represent $90^{\circ}$ pulses. Doublequantum excitation and reconversion (2Qexc, 2Qrec) can be achieved, for example, with HORROR [42] (B) or with a symmetry-based recoupling sequence, as $\mathrm{R} 18_{n}^{5}$ with $n=8$ or $n=10[46](\mathrm{C})$. The second $\mathrm{CC}$ mixing is either proton-driven spin-diffusion (D) [41] or protonmediated polarization transfer (E) $[33,34]$. Longitudinal $\left({ }^{1} \mathrm{H},{ }^{1} \mathrm{H}\right)$ mixing for proton-mediated transfer in the CCHHC experiment can be achieved by zero- or double-quantum mixing units discussed in [34]. For homonuclear CC mixing, through-bond [72] or DQ recoupling $[7,8,42,45,46]$ techniques could be used in addition to proton-driven spin-diffusion. The coherence pathway was selected by the following phase cycle: $\phi 1=\{x\}_{16}, \phi 2=\{x, y,-x,-y\}_{4}, \phi 3=\left\{x_{2},-x_{2}\right\}_{4}, \quad \phi$ $4=\left\{x_{4}, y_{4},-x_{4},-y_{4}\right\}, \quad \phi$ rec $=\{x,-x,-x, x, y,-y,-y, y,-x, x, x,-x$, $-y, y, y,-y\}$ for longitudinal CC-mixing and: $\phi 1=\{x\}_{32}, \phi 2=\left\{x_{2}\right.$, $\left.y_{2},-x_{2},-y_{2}\right\} \phi 3=\left\{x_{4},-x_{4}\right\}, \phi 4=\left\{x_{8}, y_{8},-x_{8},-y_{8}\right\}, \phi 5=\{x,-x\}_{16}$, $\phi \mathrm{rec}=\{x,-x,-x, x,-x, x, x,-x, y,-y,-y, y,-y, y, y,-y,-x, x, x,-x$, $x,-x,-x, x,-y, y, y,-y, y,-y,-y, y\}$ for proton-mediated transfer. In both cases, sign discrimination in the two indirect dimensions is accomplished by time proportional phase incrementation (TPPI) [73] of $\phi 2$ by $45^{\circ}$ with $t_{1}$, and of both phases $\phi 2$ and $\phi 3$ by $90^{\circ}$ with $t_{2}$.

tacts, and can in uniformly ${ }^{13} \mathrm{C},{ }^{15} \mathrm{~N}$-labeled proteins encode contacts between amino acid side chains separated in the primary structure, whereas dipolar $\mathrm{CC}$ mixing in uniformly labeled samples leads to a transfer between the shortest CC contacts, i.e., directly bonded carbon atoms $[33,34]$. The same set of mixing units was assumed in the context of a 3D NHHCC experiment (Fig. 2). Here, a heteronuclear magnetization transfer encodes a close contact between the protons attached directly to the nuclei involved. In contrast to standard 3D HETCOR NCC experiments [11-16], which give information about connectivities, this experiment yields information about the conformation of the protein backbone $[33,34]$.

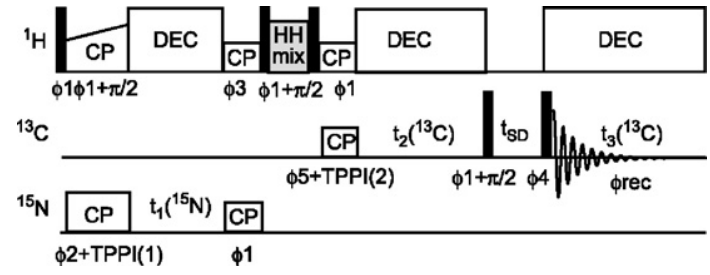

Fig. 2. Pulse sequence for the 3D heteronuclear NHHCC correlation experiment in the solid state. Black rectangles represent $90^{\circ}$ pulses. Longitudinal zero-quantum mixing was applied for $\left({ }^{1} \mathrm{H},{ }^{1} \mathrm{H}\right)$ transfer from protonated ${ }^{15} \mathrm{~N}$ to ${ }^{13} \mathrm{CH}_{x}(x=1,2,3)$ groups [33]. In addition to proton-driven spin-diffusion, through-bond [74] or DQ recoupling $[7,8,42,45,46]$ techniques could be used for homonuclear CC mixing. Indicated RF and receiver phases for the NHHCC experiments are: $\phi$ $1=\{x\}_{32}, \phi 2=\{x,-x\}_{16}, \phi 3=\left\{x_{2}, x_{2}\right\}_{8}, \phi 4=\left\{x_{8}, y_{8},-x_{8},-y_{8}\right\} \quad \phi$ $5=\left\{x_{4},-x_{4}\right\}_{4}, \phi \mathrm{rec}=\{x,-x,-x, x,-x, x, x,-x, y,-y,-y, y,-y, y, y$, $-y,-x, x, x,-x, x,-x,-x, x,-y, y, y,-y, y,-y,-y, y\}$. Sign discrimination in both indirect dimensions was achieved with TPPI of $\phi 2$ by $90^{\circ}$ with $t_{1}$, and of $\phi 4$ by $90^{\circ}$ with $t_{2}$.

Compared to [33,34], $\left({ }^{1} \mathrm{H},{ }^{1} \mathrm{H}\right)$ mixing has been implemented without an initial proton dephasing delay by setting the phases of the first two proton CP-pulses orthogonal to each other. As a result, artifacts due to $\mathrm{CP}$ interference effects between proton magnetization created before and after $t_{1}$ are minimized. Both types of correlation experiments can be easily modified if other transfer schemes, in particular at MAS rates above $15 \mathrm{kHz}[34,38]$, are preferable.

\subsection{Sensitivity considerations for multidimensional NMR experiments}

The sensitivity of a processed NMR spectrum is defined as the signal-to-noise ratio, scaled with the square root of the total number of scans, $N$ [58]. For a multidimensional spectrum, the signal, $S$, is proportional to the total number of scans and the normalized integral of the weighted time-domain signal envelope $s^{\mathrm{e}}\left(t_{1}, t_{2}, \ldots, t_{\mathrm{i}}\right)$ over the sampled multidimensional time space (Eq. (1)).

$$
\begin{aligned}
S \sim & \frac{N}{\iiint_{t_{1}, t_{2}, \ldots, t_{i}} \mathrm{~d} t_{1} \mathrm{~d} t_{2} \cdots \mathrm{d} t_{i}} \\
& \times \iiint_{t_{1}, t_{2}, \ldots, t_{i}} \mathrm{~d} t_{1} \mathrm{~d} t_{2} \cdots \mathrm{d} t_{i} s^{\mathrm{e}}\left(t_{1}, t_{2}, \ldots, t_{i}\right) \\
& \times h\left(t_{1}, t_{2}, \ldots, t_{i}\right) .
\end{aligned}
$$

On the other hand, the root mean square (rms) noise, $\sigma_{\mathrm{N}}$, is proportional to the square root of the total number of scans and the normalized rms amplitude of the weighting function $h\left(t_{1}, t_{2}, \ldots, t_{i}\right)$ (Eq. (2)):

$$
\begin{aligned}
\sigma_{N} & \sim \sqrt{\frac{N}{\iint_{t_{1}, t_{2}, \ldots, t_{i}} \mathrm{~d} t_{1} \mathrm{~d} t_{2} \cdots \mathrm{d} t_{i}} \iiint_{t_{1}, t_{2}, \ldots, t_{i}} \mathrm{~d} t_{1} \mathrm{~d} t_{2} \cdots \mathrm{d} t_{i} \cdot h^{2}\left(t_{1}, t_{2}, \ldots, t_{i}\right)} \\
& =\sqrt{N \cdot\left\langle h^{2}\right\rangle} .
\end{aligned}
$$


In a three-dimensional experiment, the length of the direct time domain, $t_{3 \max }$, does not affect the experiment time, and the signal-to-noise ratio $S / \sigma_{\mathrm{N}}$ can be optimized after the acquisition by applying an appropriate filter function in this dimension. If we further assume purely homogeneous line broadening (equivalent to a signal envelope that factorizes into a product $s^{\mathrm{e}}\left(t_{1}\right)$. $\left.s^{\mathrm{e}}\left(t_{2}\right) \cdot s^{\mathrm{e}}\left(t_{3}\right)\right)$, it is sufficient to consider the sensitivity of a three-dimensional spectrum as a function of the two-dimensional time space sampled in the indirect time domains $t_{1}$ and $t_{2}$. Assuming that the repetition rate has been optimized, the sensitivity (as defined in Eq. (4.3.17) in [58]) is given by

$$
\frac{S}{\sigma_{\mathrm{N}} \sqrt{N}} \sim \frac{\iint_{t_{1}, t_{2}} \mathrm{~d} t_{1} \mathrm{~d} t_{2} \cdot s^{\mathrm{e}}\left(t_{1}, t_{2}\right) \cdot h\left(t_{1}, t_{2}\right)}{\sqrt{\left\langle h^{2}\right\rangle} \iint_{t_{1}, t_{2}} \mathrm{~d} t_{1} \mathrm{~d} t_{2}} .
$$

The sensitivity is independent of the number of scans, depending only on the region of the $\left(t_{1}, t_{2}\right)$ space which is sampled and on the filter function, $h\left(t_{1}, t_{2}\right)$. It is well known that the filter function giving rise to maximum sensitivity is the matched filter function $h\left(t_{1}, t_{2}\right)=s^{\mathrm{e}}\left(t_{1}, t_{2}\right)$, however, often at the expense of resolution [58]. Alternatively, the sampling scheme can be adjusted to match the decay curve $s^{\mathrm{e}}\left(t_{1}, t_{2}\right)$. Apart from nonlinear sampling [59], where the density of the sampled $\left(t_{1}, t_{2}\right)$ points is proportional to $s^{\mathrm{e}}\left(t_{1}, t_{2}\right)$, or in situ filtering [60], where the number of scans per slice is proportional to $s^{\mathrm{e}}\left(t_{1}, t_{2}\right)$, the regularly sampled $\left(t_{1}, t_{2}\right)$ space can be restricted to a region where the intensity of $s^{\mathrm{e}}\left(t_{1}, t_{2}\right)$ is above a certain minimum [56,57]. This method is particularly attractive as the fast Fourier transform algorithm is still applicable. In the simplest case, the signal decays exponentially with the time constants $T_{2}^{(1)}$ and $T_{2}^{(2)}$ in the indirect time domains, giving rise to a signal envelope $s^{\mathrm{e}}\left(t_{1}, t_{2}\right)$ as described in Eq. (4) (Fig. 3A).

$s^{\mathrm{e}}\left(t_{1}, t_{2}\right)=s^{\mathrm{e}}(0,0) \cdot \exp \left\{-t_{1} / T_{2}^{(1)}\right\} \cdot \exp \left\{-t_{2} / T_{2}^{(2)}\right\}$.

The contour levels where $s^{\mathrm{e}}\left(t_{1}, t_{2}\right)=a$ are defined by the equation $t_{1} / T_{2}^{(1)}+t_{2} / T_{2}^{(2)}=\ln \left(s^{\mathrm{e}}(0,0) / a\right)$ and the $\left(t_{1}, t_{2}\right)$ space matching the region of highest intensity corresponds to a triangle in the $\left(t_{1}, t_{2}\right)$ region (Fig. 3B) [56]. The normalized integral of $s^{\mathrm{e}}\left(t_{1}, t_{2}\right)$ (without a filter function) over the full $\left(t_{1}, t_{2}\right)$ space, $\left\langle s^{\mathrm{e}}\left(t_{1}, t_{2}\right)\right\rangle$, is (with $\left.t_{1 \max } / T_{2}^{(1)}=t_{2 \max } / T_{2}^{(2)}=t_{i \max } / T_{2}^{(i)}\right)$ :

$$
\begin{aligned}
\left\langle s^{\mathrm{e}}\left(t_{1}, t_{2}\right)\right\rangle_{\text {rectangular }}= & \frac{1}{t_{1 \max } \cdot t_{2 \max }} \int_{0}^{t_{1 \max }} \mathrm{d} t_{1} \int_{0}^{t_{2 \max }} \mathrm{d} t_{2} \\
& \times \exp \left\{-t_{1} / T_{2}^{(1)}-t_{2} / T_{2}^{(2)}\right\} \\
= & \frac{T_{2}^{(i)^{2}}}{t_{i \max }^{2}}\left(1+\exp \left\{-2 t_{i_{\max }} / T_{2}^{(i)}\right\}\right. \\
& \left.-2 \exp \left\{-t_{i \max } / T_{2}^{(i)}\right\}\right) .
\end{aligned}
$$
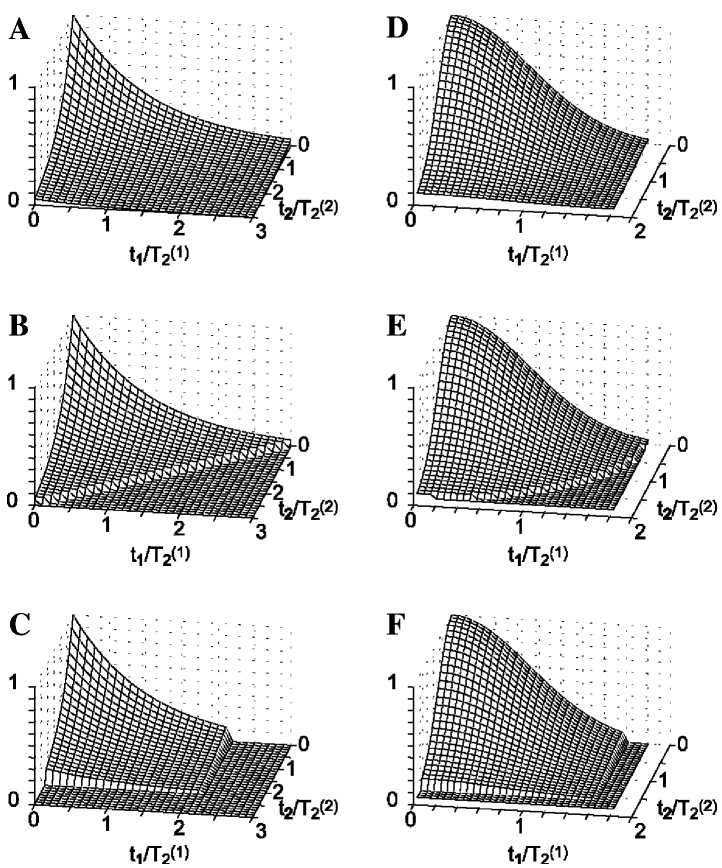

Fig. 3. (A) Two-dimensional signal envelope $s^{\mathrm{e}}\left(t_{1}, t_{2}\right)$ for signals with Lorentzian lineshape. (B) Triangular acquisition scheme for a Lorentzian signal. (C) Signal envelope $s^{\mathrm{e}}$ for a spectrum rectangularly sampled over the same area in the $\left(t_{1}, t_{2}\right)$-space as in (B). (D) Two-dimensional signal envelope $s^{\mathrm{e}}$ for signals with Gaussian lineshape. (E) Circular acquisition scheme for Gaussian signals, (F) reduced rectangle spanning the same area in the $\left(t_{1}, t_{2}\right)$ space as $(\mathrm{E})$.

For triangular sampling with $t_{1} / t_{1 \max }+t_{2} / t_{2 \max } \leqslant 1$, we find

$$
\begin{aligned}
\left\langle s^{\mathrm{e}}\left(t_{1}, t_{2}\right)\right\rangle_{\text {triangular }}= & \frac{2}{t_{1 \max } \cdot t_{2 \max }} \int_{0}^{t_{\text {max }}} \mathrm{d} t_{1} \int_{0}^{t_{2 \max }-t_{1} \cdot t_{\text {max }} / t_{1 \max }} \mathrm{d} t_{2} \\
& \times \exp \left\{-t_{1} / T_{2}^{(1)}-t_{2} / T_{2}^{(2)}\right\} \\
= & \frac{2 \cdot T_{2}^{(i)^{2}}}{t_{i \max }^{2}}\left(1-\exp \left\{-t_{i \max } / T_{2}^{(i)}\right\}-\frac{t_{i \max }}{T_{2}^{(i)}}\right. \\
& \left.\times \exp \left\{-t_{i \max } / T_{2}^{(i)}\right\}\right) .
\end{aligned}
$$

In Fig. 4A, the sensitivity gain for a triangularly sampled spectrum, with respect to a full square, is plotted as a function of $t_{i \max } / T_{2}^{(i)}$. It is obvious that, for long indirect acquisition times $t_{\text {imax }}$, the sensitivity can be doubled by sampling only the lower triangle of the $\left(t_{1}, t_{2}\right)$ space (Fig. 3B), as the acquisition time is halved and the noise is reduced by the factor $1 / 2^{1 / 2}$ (Fig. 4A, Eq. (3)) [56]. However, the sensitivity of a multidimensional NMR spectrum is likewise increased by reducing $t_{i \max }$. Therefore, it is essential to also compare the triangularly sampled spectrum to a spectrum sampled over a rectangle of the same area, defined by $t_{i \max }^{\text {square }}=t_{i \max }^{\text {triang }} / 2^{1 / 2}$, as displayed in Fig. 3C. The ratio between the sensitivities with triangular and square sampling is then: 

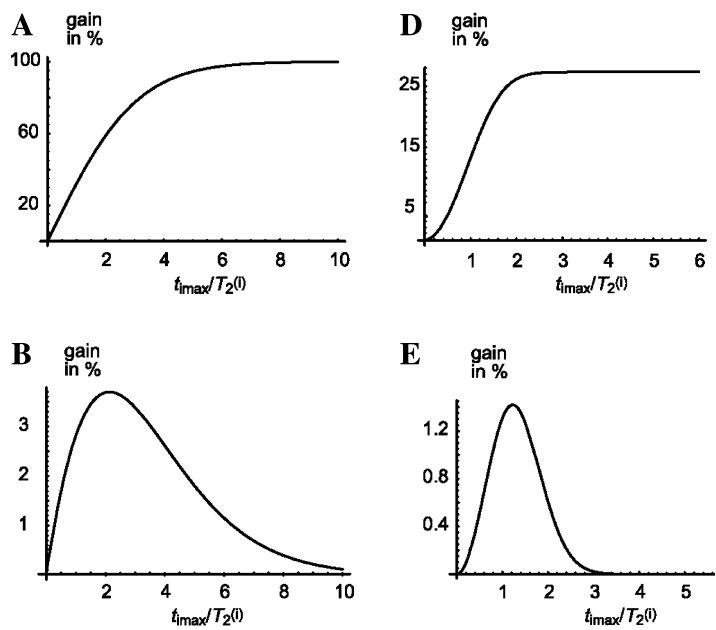

E $\quad$ in $\%$
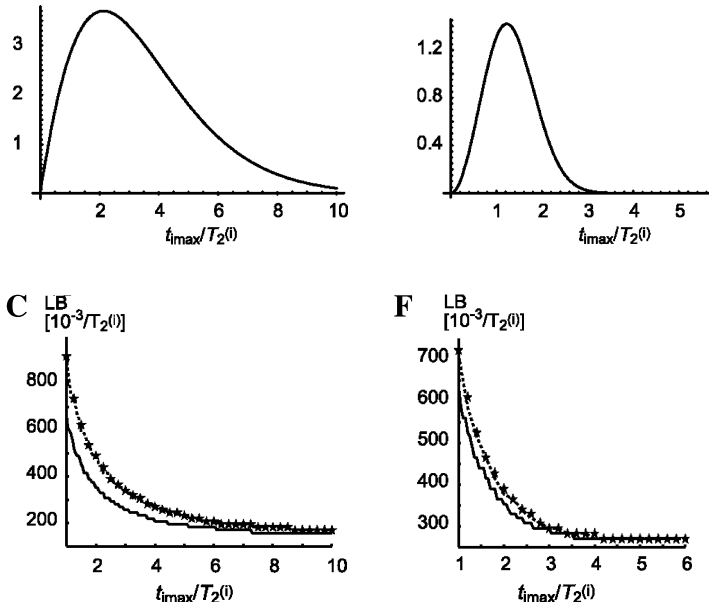

Fig. 4. (A) Sensitivity gain (in \%) for a signal with Lorentzian lineshape, if a spectrum with triangular acquisition (as in Fig. 3B) is compared to a spectrum acquired over the full rectangle (Fig. 3A), as a function of $t_{i \max } / T_{2}^{(i)}$, with $t_{i \max } / T_{2}^{(i)}=t_{1 \max } / T_{2}^{(1)}=t_{2 \max } / T_{2}^{(2)}$. (B) Sensitivity gain (in \%) for a Lorentzian signal with triangular acquisition (as in Fig. 3B) when compared to a spectrum sampled over a rectangle of the same area in the $\left(t_{1}, t_{2}\right)$-space (Fig. 3C), with $t_{i \max }^{\text {square }}=t_{i \max } / 2^{1 / 2}$ as a function of $t_{i \max } / T_{2}^{(i)}$. (C) Linewidths at half signal heights for a simulated spectrum with Lorentzian lineshape, acquired with the triangular acquisition scheme (stars) and the reduced square (dotted line) and for a spectrum acquired over the full rectangle (full line) as a function of $t_{i \max } / T_{(i)}^{2}$, with $t_{i \max } / T_{2}^{(i)}=t_{1 \max } / T_{2}^{(1)}=t_{2 \max } / T_{2}^{(2)}$. (D) Sensitivity gain (in $\%$ ) for a Gaussian signal for a spectrum with circular acquisition (as in Fig. 3E) versus a spectrum acquired over the full rectangle (Fig. 3D) as a function of $t_{i \max } / T_{2}^{(i)}$. (E) Sensitivity gain (in \%) of a Gaussian signal in a circularly sampled spectrum, compared to a spectrum sampled over the reduced square (as in Fig. 3F). (F) Linewidths at half signal heights for a simulated spectrum with Gaussian lineshape (stars), acquired with the circular acquisition scheme and the reduced square (dotted line) and for a spectrum acquired over the full rectangle (full line) as a function of $t_{i \max } / T_{2}^{(i)}$, with $t_{i \max } / T_{2}^{(i)}=t_{1 \max } / T_{2}^{(1)}=t_{2 \max } / T_{2}^{(2)}$.

Sensitivity ${ }^{\text {triangular }}$

Sensitivity ${ }^{\text {rectangular }}$

$$
=\frac{\exp \left\{(\sqrt{2}-1) \cdot t_{i \max } / T_{2}^{(i)}\right\} \cdot\left(1+t_{i \max } / T_{2}^{(i)}-\exp \left\{-t_{i \max } / T_{2}^{(i)}\right\}\right)}{\left(1-\exp \left\{-t_{i \max } / \sqrt{2} T_{2}^{(i)}\right\}\right)^{2}} .
$$

Here, $t_{i \max }$ is the maximum value in the triangular sampling scheme. In Fig. 4B, the sensitivity gain for a triangularly sampled spectrum with respect to the reduced square is plotted. A maximum intensity gain of $3.75 \%$ can be obtained for $t_{i \max } / T_{2}^{(i)}=2.3$ in the triangular sampling scheme. For long maximum acquisition times, the sensitivity becomes equal for the two cases. A reduction of the indirect time space is always accompanied by a decrease in resolution. Therefore, a thorough comparison of different acquisition schemes also has to include the linewidths in the indirect dimensions. In Fig. 4C, the linewidths in the indirect dimensions of spectra obtained with the three acquisition schemes are displayed as a function of $t_{i \max } / T_{2}^{(i)}$, obtained from numerical simulations (assuming $T_{2}^{(1)}=T_{2}^{(2)}$ ). Within the digital resolution, no difference between the linewidths at half height in $F 1$ and $F 2$ of the triangular (stars) and the reduced sampling scheme (dotted line) could be found. However, compared to the full rectangular spectrum (full line), at least in the region where the sensitivity gain is strongest, the signal width at half height is enhanced for the triangular sampling scheme. In contrast to the linewidth, the truncation artifacts depend strongly on the sampling scheme. A comparison of the simulated lineshapes obtained with $t_{i \max } / T_{2}^{(i)}=2$ with triangular and rectangular truncation is shown in Figs. 5A and B. For the reduced rectangle (Fig. 5B), the Lorentzian line exhibits the well-known orthogonal oscillatory signal tails along $F 1$ and $F 2$ [58]. The line generated with triangular acquisition (Fig. 5A) on the other hand is surrounded by orthogonal oscillations inclined by $45^{\circ}$ with respect to the $F 1$ and $F 2$ axes.

For a signal with a Gaussian lineshape, characterized by a decay with $\exp \left\{-\left(t_{1} / T_{2}^{(1)}\right)^{2}-\left(t_{2} / T_{2}^{(2)}\right)^{2}\right\}$ (Fig. 3D), the matched filtering condition is fulfilled by an elliptic
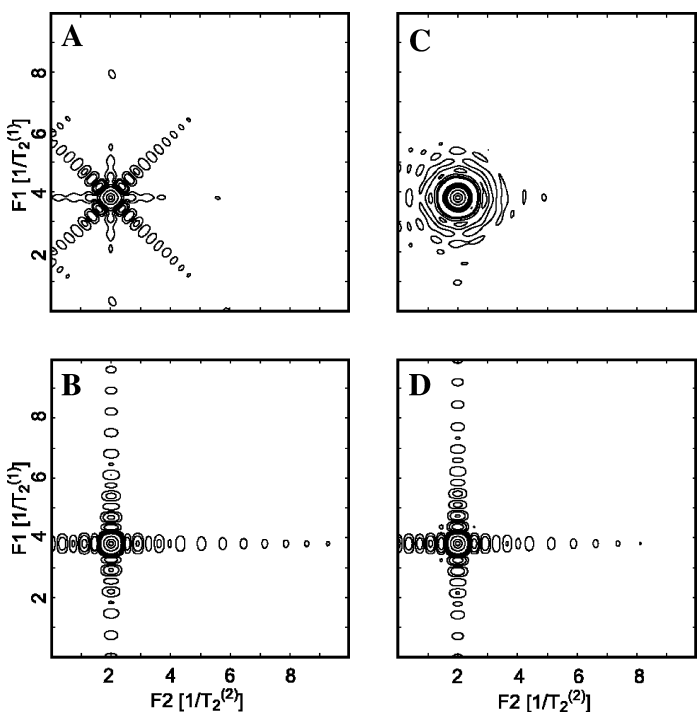

Fig. 5. (A,B) Simulated peak shapes for a Lorentzian line with $t_{i \max } / T_{2}^{(i)}=2$. (A) Triangular acquisition, (B) reduced rectangular acquisition, leading to an effective $t_{i \max } / T_{2}^{(i)}=1.41$. (C,D) Simulated peak shapes for a Gaussian line for different acquisition schemes with $t_{i \max } / T_{2}^{(i)}=1.5$. (C) Circular acquisition, (D) reduced rectangular acquisition, leading to an effective $t_{i \max } / T_{2}^{(i)}=1.18$. Contour lines were drawn at $2,5,10,20,50,80$, and $95 \%$ of the peak height. 
acquisition scheme defined by $\left(t_{1} / t_{1 \max }\right)^{2}+\left(t_{2} /\right.$ $\left.t_{2 \max }\right)^{2} \leqslant 1$ (Fig. 3E).

In this case, the normalized integral of $s^{\mathrm{e}}\left(t_{1}, t_{2}\right)$ (without a filter function) over the full $\left(t_{1}, t_{2}\right)$ space is:

$$
\begin{aligned}
\left\langle s^{\mathrm{e}}\left(t_{1}, t_{2}\right)\right\rangle_{\text {rectangular }}= & \frac{1}{t_{1 \max } \cdot t_{2 \max }} \int_{0}^{t_{1} \max } \mathrm{d} t_{1} \int_{0}^{t_{2 \max }} \mathrm{d} t_{2} \\
& \times \exp \left\{-\left(t_{1} / T_{2}^{(1)}\right)^{2}-\left(t_{2} / T_{2}^{(2)}\right)^{2}\right\} \\
= & \frac{\pi \cdot T_{2}^{(i)^{2}}}{4 t_{i \max }^{2}} \operatorname{erf}\left\{\left(t_{1} / T_{2}^{(1)}\right)^{2}\right\}
\end{aligned}
$$

and with elliptic acquisition

$$
\begin{aligned}
\left\langle s^{\mathrm{e}}\left(t_{1}, t_{2}\right)\right\rangle_{\text {elliptic }}= & \frac{\pi}{4 t_{1 \max } \cdot t_{2 \max }} \int_{0}^{t_{1 \max }} \mathrm{d} t_{1} \\
& \times \int_{0}^{\sqrt{t_{2 \max }^{2}-\left(t_{1} \cdot t_{\max } / t_{\max }\right)^{2}}} \mathrm{~d} t_{2} \\
& \times \exp \left\{-\left(t_{1} / T_{2}^{(1)}\right)^{2}-\left(t_{2} / T_{2}^{(2)}\right)^{2}\right\} \\
= & \frac{\pi \cdot T_{2}^{(i)^{2}}}{4 t_{\text {max }}^{2}}\left(1-\exp \left\{-\left(t_{i \max } / T_{2}^{(i)}\right)^{2}\right\}\right) .
\end{aligned}
$$

The sensitivity compared to the full rectangle is increased by the factor $4 / \pi$ for long indirect acquisition times $t_{i \max }$, corresponding to the ratios of the areas sampled (Fig. 4D). The maximum sensitivity gain compared to a reduced rectangle of the same area (Fig. $3 \mathrm{~F}$ ) is:

$$
\frac{\text { Sensitivity }}{\text { Sensitivity }^{\text {ellictangular }}}=\frac{1-\exp \left\{-\left(t_{i \max } / T_{2}^{(i)}\right)^{2}\right\}}{\operatorname{erf}\left\{\left(t_{i \max } \sqrt{\pi} / 2 T_{2}^{(i)}\right)^{2}\right\}} .
$$

Here, a maximum sensitivity gain of $1.3 \%$ can be obtained (Fig. 4E), with linewidths (Fig. 4F, stars) identical to those of the reduced rectangle (Fig. 4F, dotted line), whereas the linewidths with respect to the full rectangle (Fig. 4F, full line) are increased. Again, the shape of the truncation artifacts is changed from orthogonal oscillation patterns along $F 1$ and $F 2$ for square acquisition (Fig. 5D) to a circular arrangement of oscillations around the Gaussian signal (Fig. 5C) acquired with circular acquisition. Here, $t_{i \max } / T_{2}^{(i)}=1.5$ was used for the elliptic acquisition scheme.

In summary, the sensitivity of a multidimensional NMR spectrum can be increased by a few percent without loss in resolution if the sampling scheme is adjusted to the decay curve in the indirect dimensions. However, in contrast to previous findings [56,57], the resolution with respect to a spectrum obtained with traditional sampling over the same area in $\left(t_{1}, t_{2}\right)$ space does not improve upon changing the sampling scheme. Truncation artifacts are altered by the acquisition scheme. In the so- lid state, lineshapes can be further complicated by inhomogeneous contributions, which will result in the formation of echoes, and, as a consequence, deteriorate the match between the acquisition scheme and the 2D decay curve.

\section{Results and discussion}

In Fig. 6A, the CCHHC spectrum of Fmoc-U$\left[{ }^{13} \mathrm{C},{ }^{15} \mathrm{~N}\right]$ valine, obtained at $400 \mathrm{MHz}$ proton frequency with $13 \mathrm{kHz}$ MAS, is displayed. Double-quantum coherences were excited and reconverted with HORROR [42]. The three resonances in the $F 1$ dimension correspond to double-quantum coherences in the side chain region between $C \alpha$ and $C \beta$, and between $C \beta$ and each $C \gamma$. Due to the use of a weak RF field $(6.5 \mathrm{kHz})$, double-quantum
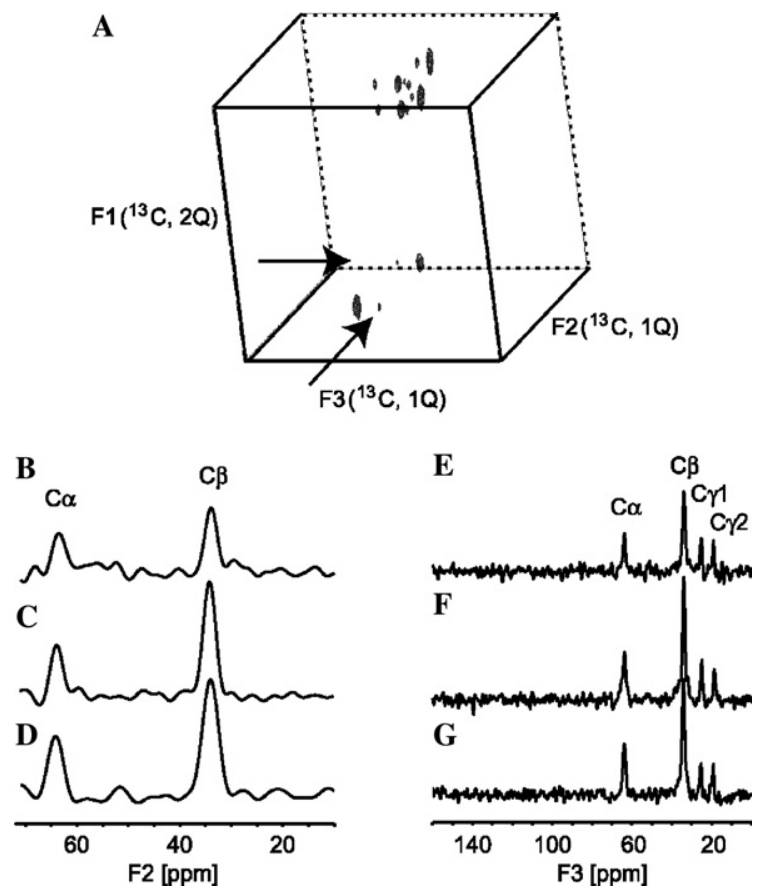

Fig. 6. (A) Homonuclear ${ }^{13} \mathrm{C} 3 \mathrm{D}$ CCHHC spectrum (pulse scheme Fig. 1A, with longitudinal $\left({ }^{1} \mathrm{H},{ }^{1} \mathrm{H}\right)$ mixing as in Fig. 1E) of Fmoc-U$\left[{ }^{15} \mathrm{~N},{ }^{13} \mathrm{C}\right]$ valine, recorded at $400 \mathrm{MHz}$ and $13 \mathrm{kHz}$ MAS. Doublequantum excitation and reconversion was achieved with HORROR [42] with excitation and reconversion time each being $154 \mu \mathrm{s}$. The longitudinal proton mixing time $t_{\mathrm{HH}}$ was $120 \mu \mathrm{s}$. The spectrum was acquired with $55 t_{1}$ and $t_{2}$ increments each, corresponding to $t_{1 \max }=t_{2 \max }=4.2 \mathrm{~ms}$. With 16 scans per slice, the total acquisition time was $27 \mathrm{~h}$. (B and E) Two 1D slices of spectrum (A), as indicated by the arrows. (C and F) Corresponding $1 \mathrm{D}$ slices of a spectrum similar to (A) obtained in the same time with triangular sampling (as in Fig. $3 \mathrm{~B}$ ) of the $t_{1}, t_{2}$ space with $t_{1 \max }=t_{2 \max }=4.2 \mathrm{~ms}$ and 32 scans per slice. In (D) and (G), the same slices from a similar spectrum, acquired with reduced square sampling (as in Fig. 3C), are displayed. For this spectrum $39 t 1$ and $t 2$ increments according to $t_{1 \max }=t_{2 \max }=3.0 \mathrm{~ms}$ were recorded with 32 scans per slice within $27 \mathrm{~h}$. All three spectra were apodized with a squared trigonometric window function Qsine3 $\left(\sin ^{2}\left\{\pi\left(1-Q^{-1}\right) t / t_{\max } \pi / Q\right\}\right.$ with $\left.Q=3\right)$ in all dimensions. 
coherences between $\mathrm{C} \alpha$ and carbonyl resonances are not created. Because of the double-quantum dimension, each peak is encoded with at least two frequencies, and the spectrum is not dominated by a cubic diagonal. Cross-peaks in the respective $F 2 / F 3$-planes indicate spatial proximity between protons attached directly to the corresponding ${ }^{13} \mathrm{C}$ atoms. Two orthogonal $1 \mathrm{D}$ slices, one parallel to $F 2$, the other parallel to $F 3$, are shown in Figs. 6B and E, respectively. Both slices are taken from the $\mathrm{C} \alpha \mathrm{C} \beta$ double-quantum $F 2 / F 3$ plane, along the $\mathrm{C} \beta$ resonance frequency in $F 3$ or $F 2$, respectively, as indicated by the arrows in Fig. 6A. The 1D slice along $F 2$ (Fig. 6B) displays the diagonal signal $\mathrm{C} \beta$ and the back-transfer peak from $\mathrm{C} \alpha$, the $1 \mathrm{D}$ slice along $F 3$ (Fig. 6E) indicates that magnetization transfer has occurred during the longitudinal proton mixing time of $120 \mu$ from $\mathrm{H} \beta$ to $\mathrm{H} \alpha$ and the $\mathrm{H} \gamma$ atoms of both methyl groups, and that these protons are therefore in close proximity. The spectrum was acquired with traditional sampling, as outlined in Fig. 3A, with $t_{1 \max }=$ $t_{2 \max }=4.2 \mathrm{~ms}$, corresponding roughly to $3 \times T_{2}$ in both indirect dimensions. For comparison, 1D slices obtained using triangular (Fig. 3B) and rectangular (Fig. 3C) truncation are displayed in Figs. 6C, D, F, and $\mathrm{G}$, respectively. The corresponding $3 \mathrm{D}$ data sets were acquired for the same total experimental time. All three spectra were apodized with a squared trigonometric window function Qsine3 $\left(\sin ^{2}\left\{\pi\left(1-Q^{-1}\right) t / t_{i \max }-\pi / Q\right\}\right.$ with $Q=3$ ) in all three dimensions and scaled to give the same noise level. Except for changes due to variations in $t_{i \max }$, the linewidths at half signal height in the indirect dimensions are similar within the limit of digital resolution $(20 \mathrm{~Hz})$ for all considered sampling schemes. The $S / \sigma_{\mathrm{N}}$ ratio is increased by a factor of roughly 1.5 for both truncated spectra with respect to the full spectrum. Although the signal is almost completely decayed in the second half of the $\left(t_{1}, t_{2}\right)$ space, the sensitivity gain upon truncation is well below 2 . This observation can be explained by the apodization, which scales down the noise acquired during the second half of the experiment.

Fig. 7A displays the homonuclear CCC spectrum of $\mathrm{U}-\left[{ }^{13} \mathrm{C},{ }^{15} \mathrm{~N}\right]$ ubiquitin. Excitation and reconversion of double-quantum coherences between directly bonded side chain carbon spins was achieved by $\mathrm{R} 18_{10}^{5}$ [46] with a mixing time of $910 \mu \mathrm{s}$. The second homonuclear mixing block consists of a spin-diffusion delay with a mixing time of $50 \mathrm{~ms}$, which is long enough to enable intra-residue magnetization transfer along the full amino acid side chain. In Fig. $7 \mathrm{~B}$, the $F 1 / F 3$ plane of the CCC spectrum at the resonance frequency of $40 \mathrm{ppm}$ in $F 2$ is displayed. This frequency corresponds to the $C \beta$ chemical shift of the amino acid I3 [61]. This slice exhibits cross-peaks between all three double-quantum coherences involving $C \beta$, i.e., $C \alpha C \beta, C \beta C \gamma 1$, and $C \beta C \gamma 2$, in $F 1$ and all resonances of the Ile residue, i.e., $\mathrm{C}^{\prime}, \mathrm{C} \alpha$, $\mathrm{C} \beta, \mathrm{C} \gamma 1, \mathrm{C} \delta 1$, and $\mathrm{C} \gamma 2$ along $F 3$, as indicated by the
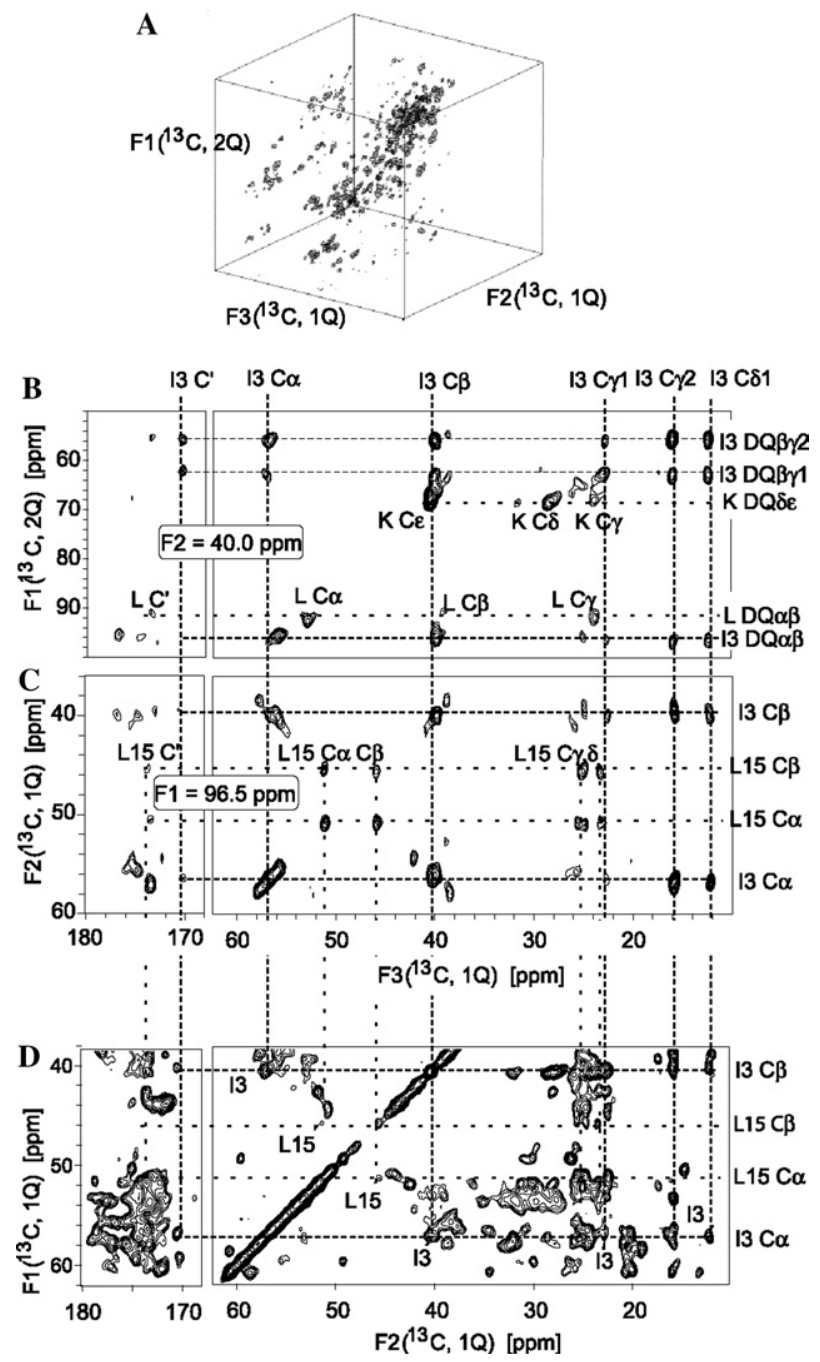

Fig. 7. (A) $3 \mathrm{D}$ CCC spectrum of $\mathrm{U}-\left[{ }^{13} \mathrm{C},{ }^{15} \mathrm{~N}\right]$ ubiquitin, obtained at $600 \mathrm{MHz}$ proton frequency with $11 \mathrm{kHz}$ MAS. The spectrum was acquired using the regular rectangular sampling scheme, with $108 t_{1}$ and $90 \quad t_{2}$ increments, corresponding to $t_{1 \max }=2.56 \mathrm{~ms}$ and $t_{2 \max }=3.73 \mathrm{~ms}$. With 16 scans per slice, the total experiment time was $90 \mathrm{~h}$. Double-quantum coherence was excited and reconverted with the $\mathrm{R} 18_{10}^{5}$ sequence with excitation and reconversion time each being $910 \mu \mathrm{s}$. Proton-driven spin-diffusion with a mixing time of $50 \mathrm{~ms}$ was applied as a second homonuclear mixing step. The spectrum was processed by multiplication with a Qsine4 window function in all three dimensions. (B) $F 1 / F 3$ plane at $F 2=40 \mathrm{ppm}$, corresponding to the $\mathrm{C} \beta$ frequency of I3. (C) $F 2 / F 3$ plane at $F 1=96.5 \mathrm{ppm}$, corresponding to the $\mathrm{C} \alpha / \mathrm{C} \beta$ double-quantum frequency of I3. Spin systems of I3 are indicated with dashed lines, spin systems of other amino acids with dotted lines. (D) 2D spin-diffusion spectrum, $600 \mathrm{MHz}$ proton frequency with $11 \mathrm{kHz}$ MAS. The mixing time was $40 \mathrm{~ms}$, and $512 t_{1}$ experiments and 16 scans per slice.

dashed lines. Furthermore, the spin system of one Lysine side chain at the $\mathrm{C} \delta \mathrm{C} \varepsilon$ double-quantum resonance and of one Leucine at the $\mathrm{C} \alpha \mathrm{C} \beta$ and double-quantum coherence can be seen (dotted line). In Fig. 7C, the $F 2 / F 3$ plane at $96.5 \mathrm{ppm}$, corresponding to the $\mathrm{C} \alpha \mathrm{C} \beta$ double-quantum coherence of $\mathrm{I} 3$, is shown. The diagonal and back-transfer (anti-diagonal) peaks for $\mathrm{C} \alpha$ and 
$\mathrm{C} \beta$ of I3 span a square in the $F 1 F 2$-plane. Two sets of true 3D signals for all other spins belonging to the $\mathrm{I} 3$ chain are clearly visible at the $\mathrm{C} \alpha$ as well as the $C \beta$ resonance in $F 2$. Apart from the signals of I3, the L15 resonances related to the $\mathrm{C} \alpha \mathrm{C} \beta$ double-quantum coherence at $96.5 \mathrm{ppm}$ (dotted line) are clearly visible. In Figs. 7D, a 2D spin-diffusion spectrum obtained with a mixing time of $40 \mathrm{~ms}$ is displayed for comparison. The resolution in the $\mathrm{C}^{\prime} / \mathrm{C} \alpha$ cross-peak region is strongly limited due to spectral overlap, caused by the rather poor spectral dispersion of $\mathrm{C} \alpha$ as well as $\mathrm{C}^{\prime}$ resonance frequencies. In contrast, in the $3 \mathrm{D}$ spectrum, each $\mathrm{C}^{\prime}$ resonance is now correlated with the $C \alpha$ as well as the $C \beta$ resonance of the corresponding amino acid, and the $\mathrm{C}^{\prime}$ signals of $\mathrm{I} 3$ and L15 can be clearly identified. The same applies for the side chain resonances $\mathrm{C} \gamma$ and $\mathrm{C} \delta$, which are now clearly separated from all other signals. If the spinning frequency is close to rotational resonance, a long spindiffusion time $(>100 \mathrm{~ms})$ can give rise to inter-residue magnetization transfer [47]. It is, therefore, straightforward to extend this CCC experiment for obtaining sequential $\left({ }^{13} \mathrm{C},{ }^{13} \mathrm{C}\right)$ resonance assignments.

In Fig. 8A, the 3D NHHCC (Fig. 2) correlation spectrum of $\mathrm{U}-\left[{ }^{13} \mathrm{C},{ }^{15} \mathrm{~N}\right]$ ubiquitin is shown. The proton-proton mixing time in this case was set to $90 \mu \mathrm{s}$. As discussed in further detail in $[34,62]$, polarization transfer for $\mathrm{NH}-\mathrm{HC}$ distances of about $2.5 \AA$ is maximized for this mixing time. As is well known from the solution state [26], the corresponding $(\mathrm{NH}, \mathrm{HC} \alpha)$ correlations are dominated by inter-residue $\mathrm{NH}^{i+1}-\mathrm{H}^{i} \mathrm{C} \alpha$ contacts from $\beta$-sheet regions of the protein under study. Intra-residue $\mathrm{NH}-\mathrm{HC} \alpha$ contacts from $\beta$-sheet regions and inter- and intraresidual $(\mathrm{NH}, \mathrm{HC} \alpha)$ correlations from $\alpha$-helical segments are attenuated, as the corresponding $\mathrm{NH}-\mathrm{HC} \alpha$ distances are above $2.9 \AA$ [62]. Incorporation of the NHHC transfer into a $3 \mathrm{D}$ experiment separates NHHC contacts according to the corresponding carbon resonance frequencies in $F 2$. Magnetization transfer to all side chain resonances during the following long spin-diffusion time correlates the resonance frequency with the carbon resonances of the whole side chain and thus allows for the identification of the corresponding amino acid. In Fig. 8B, the $\beta$-sheet stretch S65-T66-L67 from the crystal structure of ubiquitin is shown. Short interresidual $\mathrm{NH}^{i+1}-\mathrm{H}^{\mathrm{i}} \mathrm{C} \alpha$ contacts are indicated by solid arrows, the longer intra-residue $\mathrm{NH}-\mathrm{HC} \alpha$ contacts are marked with dotted arrows. In the $F 1 / F 2$ planes at a value of $F 3$ corresponding to the $\mathrm{C} \alpha$ chemical shifts of $\mathrm{S} 65$ (Fig. 8C) and T66 (Fig. 8D), the inter-residual T66N$\mathrm{S} 65 \mathrm{C} \alpha$ and $\mathrm{L} 67 \mathrm{~N}-\mathrm{T} 66 \mathrm{C} \alpha$ correlations dominate the weak intra-residue $\mathrm{N} / \mathrm{C} \alpha$ contacts of $\mathrm{S} 65$ and T66. The $F 2 / F 3$ slices (Fig. $8 \mathrm{E}-\mathrm{G}$ ) at the three ${ }^{15} \mathrm{~N}$ chemical shifts of $\mathrm{S} 65$, T66, and $\mathrm{L} 67$ in $F 1$ display the corresponding intra-residue $\mathrm{C} \alpha \rightarrow \mathrm{C} \beta \rightarrow \mathrm{C} \gamma$ correlations of the protein region under consideration and thus allow for the identification of the spin system involved. Hence, by corre-

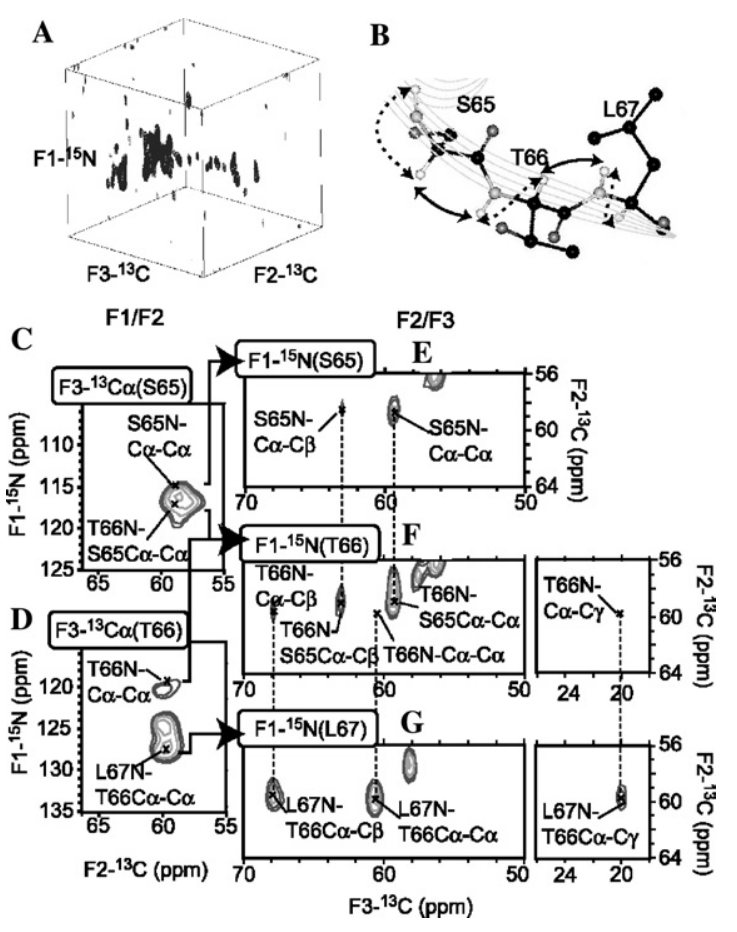

Fig. 8. (A) $3 \mathrm{D}$ NHHCC spectrum of $\mathrm{U}-\left[{ }^{13} \mathrm{C},{ }^{15} \mathrm{~N}\right]$ ubiquitin, obtained at $600 \mathrm{MHz}$ proton frequency with $11 \mathrm{kHz}$ MAS. Triangular sampling with $19 t_{1}$ and $54 t_{2}$ increments corresponding to $t_{1 \max }=2.56 \mathrm{~ms}$ and $t_{2 \max }=2.88 \mathrm{~ms}$, was applied, with 320 scans per slice, amounting to a total experiment time of 4 days. The longitudinal proton mixing time $\mathrm{t}_{\mathrm{HH}}$ was $90 \mu \mathrm{s}$, CC mixing was achieved by proton-driven spindiffusion with a mixing time of $50 \mathrm{~ms}$. The spectrum was processed by multiplication with a Qsine4 window function in the $F 1$ and with Qsine3 window functions (definition given in the text) in the $F 2$ and $F 3$ dimensions. (B) Amino acid stretch S65-L67 of ubiquitin, displaying the corresponding inter- (solid arrows) and intraresidual (dotted arrows) $\left({ }^{1} \mathrm{H},{ }^{1} \mathrm{H}\right)$ contacts. $F 1 / F 2$-planes are shown with $F 3$ corresponding to the $\mathrm{C} \alpha$ chemical shifts of $\mathrm{S} 65$ (C) and T66 (D). (E-G) F2/ $F 3$ planes at the corresponding ${ }^{15} \mathrm{~N}$ shifts in $F 1$.

lating the intra- and interresidue $\mathrm{NH}-\mathrm{HC} \alpha$ contacts with the full amino acid chain, NHHCC data yield information about the secondary structure, which complements torsion angle constraints based solely on chemical shifts.

\section{Conclusions}

We have introduced 3D pulse sequences for structure elucidation and resonance assignment of uniformly labeled proteins. Homonuclear CCC and CCHHC experiments containing one double-quantum evolution period yield 3D spectra without a cubic diagonal, such that each peak is at least encoded with two resonance frequencies. Off-diagonal peaks are encoded with three resonance frequencies, and spectral overlap is reduced with respect to a $2 \mathrm{D}$ experiment. Depending on the second mixing scheme, these spectra are useful for the identification of spin systems and sequential assignment, or 
they yield proton-proton constraints with increased spectral resolution. The NHHCC experiment allows for identification of structural elements such as $\beta$-sheets by correlating $\mathrm{NH}-\mathrm{HC}$ proton-proton contacts with the spin system of the amino acid side chain.

Furthermore, we have analyzed alternative sampling schemes in the indirect dimensions. Reducing the acquisition scheme in the indirect $\left(t_{1}, t_{2}\right)$ space to match the decay function of a 2D FID curve increases the sensitivity by a factor up to 2 for Lorentzian lines and up to $4 / \pi$ for Gaussian lines. In contrast to previous findings [56], however, this sensitivity gain is accompanied by a decrease in spectral resolution. The linewidth in both indirect dimensions of a spectrum recorded with such a reduced sampling scheme is always similar to that of a spectrum sampled over a rectangle of the same area in the $\left(t_{1}, t_{2}\right)$ space, while the sensitivity can be increased by a few percent for the lineshape adjusted sampling scheme.

\section{Experimental}

\subsection{Sample preparation}

$\mathrm{U}-\left[{ }^{15} \mathrm{~N},{ }^{13} \mathrm{C}\right] \mathrm{L}$-Valine was purchased from Cambridge Isotope Laboratories (CIL, Andover, MA). U$\left[{ }^{15} \mathrm{~N},{ }^{13} \mathrm{C}\right]$ Ubiquitin was produced recombinantly in Escherichia coli and purified according to established protocols $[63,64]$. Eight milligrams of $\mathrm{U}-\left[{ }^{15} \mathrm{~N},{ }^{13} \mathrm{C}\right] \mathrm{ubiq}-$ uitin was precipitated from PEG, as described in $[65,66]$ and packed into a $4 \mathrm{~mm}$ rotor.

\subsection{Numerical simulations}

Integrals were solved analytically with the program Mathematica, numerical simulations of the linewidths in Figs. 4C and $\mathrm{F}$ were carried out in Matlab.

Series of 2D spectra containing one cosine-modulated signal were calculated from their simulated 2D FIDs represented by following data arrays (Eq. (11)):

$$
\begin{aligned}
I(n 1, n 2)= & \cos (2 \pi \cdot n \cdot d w 1 \cdot F 1) \cos (2 \pi \cdot n \cdot d w 2 \cdot F 2) \\
& \times \operatorname{decay}(n 1, n 2),
\end{aligned}
$$

with $\operatorname{decay}(n 1, n 2)=\exp \left\{-n 1 \cdot d w^{(1)} / T_{2}^{(1)}-n 2 \cdot d w^{(2)} /\right.$ $\left.T_{2}^{(2)}\right\}$ for Lorentzian signals, and decay $(n 1, n 2)=$ $\exp \left\{-\left(n 1 \cdot d w^{(1)} / T_{2}^{(1)}\right)^{2}-\left(n 2 \cdot d w^{(1)} / T_{2}^{(1)}\right)^{2}\right\}$ for Gaussian signals. The dwell times $d w^{(i)}$ in both dimensions were set to of $0.01 \cdot T_{2}^{(i)}$, and the frequencies $F 1$ and $F 2$ were set to the arbitrary values $0.038 / d w^{(1)}$ and $0.02 /$ $d w^{(2)}$.

To compare the effect of modified sampling schemes on the linewidth depending on the acquisition length, the number of data points in both dimensions $n_{i \max }=n_{1 \max }=n_{2 \max }$ was varied from 10 to 1001 in steps of 5. Corresponding triangular (elliptic) data arrays for Lorentzian (Gaussian) signals were generated by setting all data points with $n_{1} / n_{1 \max }+n_{2} / n_{2 \max }>1$ $\left(\left(n_{1} / n_{1 \max }\right)^{2}+\left(n_{2} / n_{2 \max }\right)^{2}>1\right)$ of the full array to zero. For the corresponding reduced rectangular data arrays, $n_{i \max }$ was set to the integer closest to the square root of the total number of nonzero data points $(n 1, n 2)$ in the respective alternative sampling scheme. All $2 \mathrm{D}$ data arrays were subsequently zero-filled to 4096 points in each dimension and processed by real Fourier-transformation, yielding spectra of a size of $2048 \times 2048$ points with a resolution corresponding to $2.44 \times$ $10^{-4} / d w$. For each spectrum, the linewidth at half signal height was taken of the averaged half-widths in both dimensions.

\subsection{NMR spectroscopy}

Solid-state NMR experiments were conducted on 9.4 T $\left({ }^{1} \mathrm{H}\right.$ resonance frequency: $\left.400 \mathrm{MHz}\right)$ and $14.1 \mathrm{~T}$ $(600 \mathrm{MHz})$ wide-bore instruments (Bruker Biospin/Germany) using standard triple-resonance $\left({ }^{1} \mathrm{H},{ }^{13} \mathrm{C}\right.$, and $\left.{ }^{15} \mathrm{~N}\right)$ MAS probe heads. The sample temperatures were actively controlled at $15^{\circ} \mathrm{C}$ (valine) or $-10{ }^{\circ} \mathrm{C}$ (ubiquitin) during the course of the experiments. For initial excitation of rare-spin nuclei, Hartmann-Hahn crosspolarization $[67,68]$ with amplitude modulation on the proton RF field $[69,70]$ was used. During evolution and detection, TPPM [71] decoupling with an RF field of $83 \mathrm{kHz}$ was applied to protons. RF and receiver phases indicated in Figs. 1 and 2 are given in the respective figure captions. Signals were referenced to an external sample of solid adamantane as a secondary standard with $\delta\left(\mathrm{CH}_{2}\right)=29.5 \mathrm{ppm}$.

\section{Acknowledgments}

We thank Dr. H. Förster and Dr. M. Ackermann (Bruker/Germany) for helpful discussions, K. Giller, Dr. S. Luca, and B. Angerstein for help with the sample preparation, Dr. C.E. Hughes for carefully proofreading the manuscript, and the Stiftung Stipendien-Fonds of the FCI for a Liebig fellowship to H.H.

\section{References}

[1] E.R. Andrew, A. Bradbury, R.G. Eades, Nuclear magnetic resonance spectra from a crystal rotated at high speed, Nature 182 (1958) 1659

[2] L.K. Thompson, Solid-state NMR studies of the structure and mechanisms of proteins, Curr. Opin. Struct. Biol. 12 (2002) 661669.

[3] R. Tycko, Applications of solid state NMR to the structural characterization of amyloid fibrils: methods and results, Prog. Nucl. Magn. Reson. Spectrosc. 42 (2003) 53-68.

[4] S. Luca, H. Heise, M. Baldus, High-resolution solid-state NMR applied to polypeptides and membrane proteins, Acc. Chem. Res. 36 (2003) 858-865. 
[5] S. Luca, J.F. White, A.K. Sohal, D.V. Filippov, J.H. van Boom, R. Grisshammer, M. Baldus, The conformation of neurotensin bound to its G protein-coupled receptor, Proc. Natl. Acad. Sci. USA 100 (2003) 10706-10711.

[6] C.P. Jaroniec, C.E. MacPhee, V.S. Bajaj, M.T. McMahon, C.M. Dobson, R.G. Griffin, High-resolution molecular structure of a peptide in an amyloid fibril determined by magic angle spinning NMR spectroscopy, Proc. Natl. Acad. Sci. USA 101 (2004) 711716.

[7] R.G. Griffin, Dipolar recoupling in MAS spectra of biological solids, Nat. Struct. Biol. 5 (1998) 508-512.

[8] S. Dusold, A. Sebald, Dipolar recoupling under MAS, Annu. Rep. NMR Spectrosc. 41 (2000) 185-264.

[9] M.H. Levitt, Symmetry-based pulse sequences in magic-angle spinning solid-state NMR, in: R.K. Harris (Ed.), Encyclopedia of Nuclear Magnetic Resonance: Supplementary Volume, Wiley, Chichester, 2002, pp. 165-196.

[10] M. Baldus, Correlation experiments for assignment and structure elucidation of immobilized polypeptides under magic angle spinning, Prog. Nucl. Magn. Reson. Spectrosc. 41 (2002) 1-47.

[11] B.Q. Sun, C.M. Rienstra, P.R. Costa, J.R. Williamson, R.G. Griffin, 3D N-15-C-13-C-13 chemical shift correlation spectroscopy in rotating solids, J. Am. Chem. Soc. 119 (1997) 8540-8546.

[12] M. Hong, Resonance assignment of $13 \mathrm{C} / 15 \mathrm{~N}$ labeled solid proteins by two- and three-dimensional MAS NMR, J. Biomol. NMR 15 (1999) 1-14.

[13] C.M. Rienstra, M. Hohwy, M. Hong, R.G. Griffin, 2D and 3D N-15-C-13-C-13 NMR chemical shift correlation spectroscopy of solids: assignment of MAS spectra of peptides, J. Am. Chem. Soc. 122 (2000) 10979-10990.

[14] A. Detken, E.H. Hardy, M. Ernst, M. Kainosho, T. Kawakami, S. Aimoto, B.H. Meier, Methods for sequential resonance assignment in solid, uniformly C-13, N-15 labelled peptides: quantification and application to antamanide, J. Biomol. NMR 20 (2001) 203-221.

[15] F. Castellani, B.J. van Rossum, A. Diehl, K. Rehbein, H. Oschkinat, Determination of solid-state NMR structures of proteins by means of three-dimensional N-15-C-13-C-13 dipolar correlation spectroscopy and chemical shift analysis, Biochemistry 42 (2003) 11476-11483.

[16] T.I. Igumenova, A.J. Wand, A.E. McDermott, Assignment of the backbone resonances for microcrystalline ubiquitin, J. Am. Chem. Soc. 126 (2004) 5323-5331.

[17] N.S. Astrof, C.E. Lyon, R.G. Griffin, Triple resonance solid state NMR experiments with reduced dimensionality evolution periods, J. Magn. Reson. 152 (2001) 303-307.

[18] T. Fujiwara, Y. Todokoro, H. Yanagishita, M. Tawarayama, T. Kohno, K. Wakamatsu, H. Akutsu, Signal assignments and chemical-shift structural analysis of uniformly 13C, 15N-labeled peptide, mastoparan-X, by multidimensional solid-state NMR under magic-angle spinning, J. Biomol. NMR 28 (2004) 311-325.

[19] B.J. van Rossum, F. Castellani, K. Rehbein, J. Pauli, H. Oschkinat, Assignment of the nonexchanging protons of the alpha-spectrin SH3 domain by two- and three-dimensional H-1-C13 solid-state magic-angle spinning NMR and comparison of solution and solid-state proton chemical shifts, Chembiochem 2 (2001) 906-914.

[20] D. Sakellariou, A. Lesage, L. Emsley, Proton-proton constraints in powdered solids from H-1-H-1-H-1 and H-1-H-1-C-13 threedimensional NMR chemical shift correlation spectroscopy, J. Am. Chem. Soc. 123 (2001) 5604-5605.

[21] B.J. van Rossum, F. Castellani, J. Pauli, K. Rehbein, J. Hollander, H.J.M. de Groot, H. Oschkinat, Assignment of amide proton signals by combined evaluation of $\mathrm{HN}, \mathrm{NN}$ and HNCA MASNMR correlation spectra, J. Biomol. NMR 25 (2003) 217-223.

[22] C.M. Rienstra, M. Hohwy, L.J. Mueller, C.P. Jaroniec, B. Reif, R.G. Griffin, Determination of multiple torsion-angle constraints in U-C-13,N-15-labeled peptides: 3D H-1-N-15-C-13-H-1 dipolar chemical shift NMR spectroscopy in rotating solids, J. Am. Chem. Soc. 124 (2002) 11908-11922.

[23] V. Ladizhansky, C.P. Jaroniec, A. Diehl, H. Oschkinat, R.G. Griffin, Measurement of multiple psi torsion angles in uniformly C-13,N-15-labeled alpha-spectrin SH3 domain using 3D N-15-C13-C-13-N-15 MAS dipolar-chemical shift correlation spectroscopy, J. Am. Chem. Soc. 125 (2003) 6827-6833.

[24] R. Ramachandran, V. Ladizhansky, V.S. Bajaj, R.G. Griffin, C13-C-13 rotational resonance width distance measurements in uniformly C-13-labeled peptides, J. Am. Chem. Soc. 125 (2003) $15623-15629$.

[25] V. Ladizhansky, R.G. Griffin, Band-selective carbonyl to aliphatic side chain C-13-C-13 distance measurements in U-C-13,N-15labeled solid peptides by magic angle spinning NMR, J. Am. Chem. Soc. 126 (2004) 948-958.

[26] K. Wüthrich, NMR of Proteins and Nucleic Acids, Wiley Interscience, New York, 1986.

[27] K. Wüthrich, The way to NMR structures of proteins, Nat. Struct. Biol. 8 (2001) 923-925.

[28] X.L. Wu, S.M. Zhang, X.W. Wu, 2-Stage feature of HartmannHahn cross relaxation in magic-angle sample spinning, Phys. Rev. B 37 (1988) 9827-9829.

[29] S. Zhang, B.H. Meier, R.R. Ernst, Polarization echoes in NMR, Phys. Rev. Lett. 69 (1992) 2149-2151.

[30] M. Tomaselli, S. Hediger, D. Suter, R.R. Ernst, Nuclear magnetic resonance polarization and coherence echoes in static and rotating solids, J. Chem. Phys. 105 (1996) 10672-10681.

[31] F.M. Mulder, W. Heinen, M. van Duin, J. Lugtenburg, H.J.M. de Groot, Spin diffusion with C-13 selection and detection for the characterization of morphology in labeled polymer blends with MAS NMR, J. Am. Chem. Soc. 120 (1998) 12891-12894.

[32] M. Wilhelm, H. Feng, U. Tracht, H.W. Spiess, 2D CP/MAS C-13 isotropic chemical shift correlation established by H-1 spin diffusion, J. Magn. Reson. 134 (1998) 255-260.

[33] A. Lange, S. Luca, M. Baldus, Structural constraints from proton-mediated rare-spin correlation spectroscopy in rotating solids, J. Am. Chem. Soc. 124 (2002) 9704-9705.

[34] A. Lange, K. Seidel, L. Verdier, S. Luca, M. Baldus, Analysis of proton-proton transfer dynamics in rotating solids and their use for 3D structure determination, J. Am. Chem. Soc. 125 (2003) $12640-12648$.

[35] Y. Matsuki, H. Akutsu, T. Fujiwara, Precision H-1-H-1 distance measurement via C-13 NMR signals: utilization of H-1-H-1 double-quantum dipolar interactions recoupled under magic angle spinning conditions, Magn. Reson. Chem. 42 (2004) 291-300.

[36] R. Tycko, Y. Ishii, Constraints on supramolecular structure in amyloid fibrils from two-dimensional solid-state NMR spectroscopy with uniform isotopic labeling, J. Am. Chem. Soc. 125 (2003) 6606-6607.

[37] I. de Boer, J. Matysik, M. Amakawa, S. Yagai, H. Tamiaki, A.R. Holzwarth, H.J.M. de Groot, MAS NMR structure of a microcrystalline Cd-bacteriochlorophyll d analogue, J. Am. Chem. Soc. 125 (2003) 13374-13375.

[38] M. Etzkorn, A. Böckmann, A. Lange, M. Baldus, Probing molecular interfaces using 2D magic-angle-spinning NMR on protein mixtures with different uniform labeling, J. Am. Chem. Soc. 26 (2004) 14746-14751.

[39] K. Seidel, M. Etzkorn, L. Sonnenberg, A. Sebald, M. Baldus, Studying 3D structure and dynamics by high-resolution solid-state NMR: application to L-tyrosine-ethylester (2004) submitted.

[40] A. Lange, S. Becker, K. Seidel, O. Pongs, M. Baldus, A general concept for protein structure determination using Magic-AngleSpinning NMR: application to Kaliotoxin (2004) submitted.

[41] N. Bloembergen, On the interaction of nuclear spins in a crystalline lattice, Physica 15 (1949) 386-426. 
[42] N.C. Nielsen, H. Bildsoe, H.J. Jakobsen, M.H. Levitt, Doublequantum homonuclear rotary resonance-efficient dipolar recovery in magic-angle-spinning nuclear-magnetic-resonance, J. Chem. Phys. 101 (1994) 1805-1812.

[43] M. Hong, R.G. Griffin, Resonance assignments for solid peptides by dipolar-mediated C-13/N-15 correlation solid-state NMR, J. Am. Chem. Soc. 120 (1998) 7113-7114.

[44] M. Baldus, A.T. Petkova, J. Herzfeld, R.G. Griffin, Cross polarization in the tilted frame: assignment and spectral simplification in heteronuclear spin systems, Mol. Phys. 95 (1998) $1197-$ 1207.

[45] A. Brinkmann, M. Eden, M.H. Levitt, Synchronous helical pulse sequences in magic-angle spinning nuclear magnetic resonance: double quantum recoupling of multiple-spin systems, J. Chem. Phys. 112 (2000) 8539-8554.

[46] P.E. Kristiansen, D.J. Mitchell, J.N.S. Evans, Double-quantum dipolar recoupling at high magic-angle spinning rates, J. Magn. Reson. 157 (2002) 253-266.

[47] K. Seidel, A. Lange, S. Becker, C.E. Hughes, H. Heise, M. Baldus, Protein solid-state NMR resonance assignments from (13C,13C) correlation spectroscopy, Phys. Chem. Chem. Phys. 6 (2004) 5090-5093.

[48] L. Frydman, T. Scherf, A. Lupulescu, The acquisition of multidimensional NMR spectra within a single scan, Proc. Natl. Acad. Sci. USA 99 (2002) 15858-15862.

[49] S. Kim, T. Szyperski, GFT NMR, a new approach to rapidly obtain precise high-dimensional NMR spectral information, J. Am. Chem. Soc. 125 (2003) 1385-1393.

[50] E. Kupce, R. Freeman, Projection-reconstruction of three-dimensional NMR spectra, J. Am. Chem. Soc. 125 (2003) 13958-13959.

[51] E. Kupce, R. Freeman, Fast multi-dimensional Hadamard spectroscopy, J. Magn. Reson. 163 (2003) 56-63.

[52] T. Szyperski, G. Wider, J.H. Bushweller, K. Wüthrich, Reduced dimensionality in triple-resonance NMR experiments, J. Am. Chem. Soc. 115 (1993) 9307-9308.

[53] S. Luca, M. Baldus, Enhanced spectral resolution in immobilized peptides and proteins by combining chemical shift sum and difference spectroscopy, J. Magn. Reson. 159 (2002) 243-249.

[54] J. Leppert, B. Heise, O. Ohlenschläger, M. Görlach, R. Ramachandran, Triple resonance MAS NMR with $(13 \mathrm{C}, 15 \mathrm{~N})$ labelled molecules: reduced dimensionality data acquisition via $13 \mathrm{C}-15 \mathrm{~N}$ heteronuclear two-spin coherence transfer pathways, J. Biomol. NMR 28 (2004) 157-164.

[55] D. Rovnyak, C. Filip, B. Itin, A.S. Stern, G. Wagner, R.G. Griffin, J.C. Hoch, Multiple-quantum magic-angle spinning spectroscopy using nonlinear sampling, J. Magn. Reson. 161 (2003) $43-55$.

[56] K. Aggarwal, M.A. Delsuc, Triangular sampling of multidimensional NMR data sets, Magn. Reson. Chem. 35 (1997) 593-596.

[57] P. Blümler, B. Blümich, Triangular zero-setting in 2d spectroscopy, J. Magn. Reson. 86 (1990) 618-621.

[58] R.R. Ernst, G. Bodenhausen, A. Wokaun, Principles of Nuclear Magnetic Resonance in One and Two Dimensions, Clarendon Press, Oxford, 1987.
[59] J.C.J. Barna, E.D. Laue, M.R. Mayger, J. Skilling, S.J.P. Worrall, Exponential sampling, an alternative method for sampling in twodimensional NMR experiments, J. Magn. Reson. 73 (1987) 69-77.

[60] M.H. Levitt, R. Freeman, Simplification of NMR-spectra by masking in a 2nd frequency dimension, J. Magn. Reson. 39 (1980) 533-538.

[61] T.I. Igumenova, A.E. McDermott, K.W. Zilm, R.W. Martin, E.K. Paulson, A.J. Wand, Assignments of carbon NMR resonances for microcrystalline ubiquitin, J. Am. Chem. Soc. 126 (2004) 6720-6727.

[62] M. Etzkorn, S. Becker, A. Böckmann, M. Baldus (2004) in preparation.

[63] G.A. Lazar, J.R. Desjarlais, T.M. Handel, De novo design of the hydrophobic core of ubiquitin, Protein Sci. 6 (1997) 1167-1178.

[64] J. You, R.E. Cohen, C.M. Pickart, Construct for high-level expression and low misincorporation of lysine for arginine during expression of pET-encoded eukaryotic proteins in Escherichia coli, Biotechniques 27 (1999) 950-954.

[65] A. Böckmann, A. Lange, A. Galinier, S. Luca, N. Giraud, M. Juy, H. Heise, R. Montserret, F. Penin, M. Baldus, Solid state NMR sequential resonance assignments and conformational analysis of the $2 \times 10.4 \mathrm{kDa}$ dimeric form of the Bacillus subtilis protein Crh, J. Biomol. NMR 27 (2003) 323-339.

[66] R.W. Martin, K.W. Zilm, Preparation of protein nanocrystals and their characterization by solid state NMR, J. Magn. Reson. 165 (2003) 162-174.

[67] S.R. Hartmann, E.L. Hahn, Nuclear double resonance in rotating frame, Phys. Rev. 128 (1962) 2042-2053.

[68] A. Pines, M.G. Gibby, J.S. Waugh, Proton-enhanced NMR of dilute spins in solids, J. Chem. Phys. 59 (1973) 569-590.

[69] S. Hediger, B.H. Meier, R.R. Ernst, Cross-polarization under fast magic-angle sample-spinning using amplitude-modulated spinlock sequences, Chem. Phys. Lett. 213 (1993) 627-635.

[70] G. Metz, X.L. Wu, S.O. Smith, Ramped-amplitude cross-polarization in magic-angle-spinning NMR, J. Magn. Reson. Ser. A 110 (1994) 219-227.

[71] A.E. Bennett, C.M. Rienstra, M. Auger, K.V. Lakshmi, R.G. Griffin, Heteronuclear decoupling in rotating solids, J. Chem. Phys. 103 (1995) 6951-6958.

[72] M. Baldus, B. Meier, Total correlation in the solid state. The use of scalar couplings to determine the through-bond connectivity, J. Magn. Reson. A 121 (1996) 65-69.

[73] G. Bodenhausen, H. Kogler, R.R. Ernst, Selection of coherencetransfer pathways in NMR pulse experiments, J. Magn. Reson. 58 (1984) 370-388.

[74] M. Baldus, R.J. Iuliucci, B.H. Meier, Probing through-bond connectivities and through-space distances in solids by magicangle-spinning nuclear magnetic resonance, J. Am. Chem. Soc. 119 (1997) 1121-1124.

[75] E.K. Paulson, C.R. Morcombe, V. Gaponenko, B. Dancheck, R.A. Byrd, K.W. Zilm, High-sensitivity observation of dipolar exchange and NOEs between exchangeable protons in proteins by 3D solid-state NMR spectroscopy, J. Am. Chem. Soc. 125 (2003) 14222-14223. 